\title{
L'augmentation de l'âge de la retraite et la lutte contre la précarité financière des personnes en âge de pré-retraite
}

\section{Elena Serebryakova}

\section{(2) OpenEdition}

Journals

Édition électronique

URL : https://journals.openedition.org/rdctss/1686

DOI : $10.4000 /$ rdctss. 1686

ISSN : 2262-9815

Éditeur

Centre de droit comparé du travail et de la sécurité sociale

Édition imprimée

Date de publication : 1 avril 2019

Pagination : 206-209

ISSN : 2117-4350

\section{Référence électronique}

Elena Serebryakova, «L'augmentation de l'âge de la retraite et la lutte contre la précarité financière des personnes en âge de pré-retraite », Revue de droit comparé du travail et de la sécurité sociale [En ligne],

1 | 2019, mis en ligne le 01 novembre 2021, consulté le 13 novembre 2021. URL : http://

journals.openedition.org/rdctss/1686; DOI : https://doi.org/10.4000/rdctss.1686

\section{(c) (†) $\odot$}

Revue de droit comparé du travail et de la sécurité sociale est mise à disposition selon les termes de la Licence Creative Commons Attribution - Pas d'Utilisation Commerciale - Pas de Modification 4.0 International. 


\section{ELENA SEREBRYAKOVA}

UNIVERSITÉ NATIONALE DE RECHERCHE «ÉCOLE DES HAUTES ÉTUDES

EN SCIENCES ÉCONOMIQUES »

\section{L'AUGMENTATION DE L'ÂGE DE LA RETRAITE ET LA LUTTE CONTRE LA PRÉCARITÉ FINANCIĖRE DES PERSONNES EN ÂGE DE PRÉ-RETRAITE}

La loi, la plus impopulaire de toutes ces dernières années, est entrée en vigueur le $1^{\text {er }}$ janvier 2019. II s'agit de la réforme des retraites. La Loi ${ }^{\circ} 350-\mathrm{FZ}^{1}$ prévoit l'augmentation progressive de l'âge de départ à la retraite, qui, d'ici 2023, passera de 55 à 60 ans pour les femmes et de 60 à 65 ans pour les hommes. La réforme impacte également les pensions de retraite anticipée pour les enseignants, les médecins, les travailleurs « créatifs » (toutes formes de spectacle vivant) et les travailleurs des régions de l'Extrême Nord.

Jusqu'en 2019, les enseignants justifiant de 25 années de service, ainsi que les médecins ayant exercé leur activité pendant au moins 25 années en milieu rural ou 30 années dans les villes ou certains travailleurs «créatifs" parmi lesquels les trapézistes de cirque, danseurs de ballet et cascadeurs, pouvaient faire valoir leur droit à la retraite quel que soit leur âge. Dorénavant, ce droit ne peut plus être exercé dans les mêmes conditions, et l'assuré doit attendre 12 mois (en 2019) à 60 mois (à partir de 2023) après avoir rempli les conditions légales de durée d'activité professionnelle pour obtenir sa retraite anticipée sans condition d'âge. Les travailleurs de l'Extrême Nord et les artistes, comme par exemple, les acteurs de théâtre dramatique devront partir en retraite plus tard qu'avant, leur âge légal de départ à la retraite augmentant progressivement de 5 ans d'ici 2023.

Au cours des deux prochaines années, tous les assurés concernés pourront bénéficier d'une retraite six mois avant la date prévue par la loi. Par exemple, une femme qui atteint l'âge de 55 ans en 2019 pourra percevoir sa pension à l'âge de 55 ans et 6 mois (non 56 ans), un enseignant ou une ballerine ayant acquis 25 ans d'ancienneté totale en 2019 auront le droit de la percevoir six et non 12 mois après avoir atteint le nombre légal d'années d'activité.

En tout état de cause, les assurés réunissant les conditions pour bénéficier d'une retraite avant le 1er janvier 2019 conservent la possibilité de liquider leur pension.

La majorité des régimes de retraite anticipée n'a pas été impacté par la réforme. Parmi celles-ci, les pensions pour les mères ayant élevé 3 enfants et plus jusqu'à l'âge de 8 ans, celle pour personnes malvoyantes ou atteintes par le nanisme hypophysaire, ou pour les assurés exerçant un travail intense ou pénible, comme le personnel navigant, les sapeurspompiers et les conducteurs des bus.

1 Федеральный закон от 03.10.2018 n 350-Ф3 «О внесении изменений в отАельные законодательные акты Российской Федерации по вопросам назначения и выплаты пенсий» 
Selon le gouvernement, l'augmentation de l'âge de départ à la retraite doit permettre de revaloriser les pensions afin d'atteindre le niveau établi par la Convention n 102 de l'OIT concernant la sécurité sociale (norme minimum) ratifiée par une loi adoptée simultanément avec la Loi n 350-FZ.

L'impopularité de la réforme est liée au fait que la durée de vie des hommes reste faible. En 2016, leur espérance de vie à la naissance ne dépassait pas 65 ans dans 39 des $85^{2}$ régions. Dans ces conditions, l'augmentation de l'âge de départ à la retraite signifie qu'un nombre important d'assurés ne pourra pas en profiter. De l'avis des experts, d'ici 2035 près de 1,5 millions de personnes qui auraient pu partir à la retraite dans l'ancien dispositif ne parviendront pas à l'âge de la retraite requis par la nouvelle loi ${ }^{3}$.

Un autre argument avancé contre le recul de l'âge de départ à la retraite soulevé par les opposants de la réforme est la situation actuelle sur le marché du travail. Trouver un emploi après 45 ans est de plus en plus difficile. Les seniors de 45 à 60 ans représentaient plus d'un quart des sans-emploi du pays en $2017^{4}$. Tout en augmentant l'âge de la retraite, l'Etat a annoncé des mesures supplémentaires afin d'assurer l'emploi pour les seniors et améliorer leurs conditions de chômage.

\section{1 - DES « GARANTIES SUPPLÉMENTAIRES » D'AIDE SOCIALE AUX CHÔMEURS À L'ÂGE DE LA PRÉ-RETRAITE}

Dans la Loi sur l'emploi, les critères de «l'âge de pré-retraite » ont été modifiés par la Loi n 350-FZ. Avant 2019, cet âge a été défini comme la période de 2 ans précédant l'âge légal de la retraite. Actuellement, la durée de la période de pré-retraite est de 5 ans avant cet âge. Par exemple, l'âge légal de la retraite en 2019 est fixé à 56 ans pour les femmes et à 61 ans pour les hommes, par conséquent l'âge de la pré-retraite en 2019 est de 51 à 55 ans pour les femmes et de 56 à 60 ans pour les hommes.

L'analyse des nouveaux dispositifs législatifs montre qu'aucune mesure n'a été prise contre la précarité des chômeurs en âge de pré-retraite. La plupart des droits de ces chômeurs ont été conservés. Pour autant, les conditions d'indemnisation du chômage pour les personnes plus jeunes ont été sérieusement modifiées. Par exemple, leur durée d'indemnisation a été écourtée :

- de 6 à 3 mois pendant une période de 12 mois calendaires consécutifs pour les personnes licenciées pour motif disciplinaire ou qui n'ayant pas travaillé pendant les 12 mois précédents leur inscription au chômage, y compris ceux en recherche d'un premier emploi;

- de 12 mois pendant une période de 18 mois calendaires consécutifs à 6 mois pendant une période de 12 mois calendaires consécutifs pour les autres catégories de chômeurs.

La durée de base d'indemnisation du chômage pour les personnes en âge de préretraite hormis celles mentionnées ci-dessous, reste fixée à 12 mois pendant une période de 18 mois calendaires consécutifs. Les chômeurs de cette catégorie conservent leur droit d'allongement de la période d'indemnisation dont la durée est prolongée, au-delà 12 mois, de deux semaines complémentaires pour chaque année excédant la durée de

2 Russian statistical yearbook 2017 - Federal State Statistic service (Rosstat). - Moscow. - 2017. P. 85-86.

3 Саневич М. ГАе Аеньги? Сколько выиграет государство от повышения пенсионного возраста.

4 Russian statistical yearbook 2017. - Federal State Statistic service (Rosstat). - Moscow. - 2017. p.124. 
cotisation retraite de 20 ans pour une femme et de 25 ans pour un homme. Ils peuvent aussi, comme précédemment, bénéficier de la retraite anticipée s'ils justifient de la période de cotisation définie ci-dessus. Ce droit n'est utilisable que durant les deux ans avant l'âge légal de départ à la retraite du fait de l'impossibilité de retourner au travail.

Cependant, les chômeurs en âge de pré-retraite, comme les autres, ont perdu le droit au renouvellement de l'indemnisation du chômage. Ce droit a été assuré jusqu'au 31 décembre 2018. Selon le paragraphe 5 de l'article 31 de la Loi sur l'emploi, la période d'indemnisation de 12 mois du chômage pouvait être renouvelée si le chômeur n'avait pas trouvé d'emploi. La durée totale de l'indemnisation ne pouvait pas dépasser 24 mois pendant une période de 36 mois calendaires consécutifs. Ce dispositif n'est plus en vigueur.

Les règles de calcul de l'allocation chômage pour les personnes de l'âge de la préretraite n'ont pas été modifiées. Une personne ayant travaillé 26 semaines ou plus pendent 12 mois précédant la période de chômage percevra, au début, 75 \% de son salaire mensuel de référence. Au bout de trois mois son allocation sera réduite de $15 \%$. A partir du huitième mois, le taux de replacement est fixé à $45 \%$. Le salaire de référence prend en compte tous les salaires bruts perçus au cours des 3 derniers mois de travail, primes comprises, mais hors indemnités des périodes non travaillées comme par exemple des congés ou des arrêts de maladie.

En tout état de cause, le montant de l'allocation ne doit pas être en dessous du minimum fixé par le gouvernement.

A partir du $1^{\text {er }}$ janvier 2019, les montants minima et maxima d'indemnité chômage destinés aux personnes à l'âge de la pré-retraite peuvent être différents de ceux établis pour les autres catégories de chômeurs. Pour l'année 2019, l'allocation minimale est fixée à 1500 roubles $^{5}$ (environ 19 euros) par mois pour tous.

Selon les estimations du Ministère du travail, environ 40 \% des chômeurs de l'âge de la pré-retraite ${ }^{6}$ percevront l'allocation minimale en 2019, parmi eux des personnes ne justifiant pas de 26 semaines de travail pendant 12 mois précédant la période de chômage.

Le plafond d'indemnisation chômage en 2019 est plus élevé pour les demandeurs

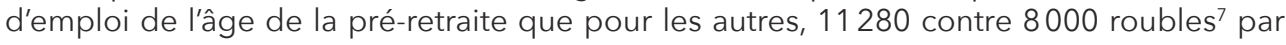
mois (environ 140 contre 100 euros). Cependant, les personnes de cet âge inscrites au Service de l'emploi avant le $1^{\text {er }}$ janvier 2019 ne peuvent bénéficier de cet avantage, le montant de leur allocation reste plafonné dans le cadre de l'ancien dispositif.

Par ailleurs, la Loi n³50-FZ aggrave la situation de certaines catégories de chômeurs de l'âge de la pré-retraite. II s'agit des personnes qui (1) n'ont pas eu d'emplois pendant 12 mois précédant leur chômage ; (2) ont été licenciées pour motif disciplinaire ; (3) ont été renvoyées de la formation prescrite par le Service de l'emploi. La durée de leur indemnité

5 Voir : Постановление Правительства РФ от 15.11.2018 n 1375 « размерах минимальной и максимальной величин пособия по безработице на 2019 год"

http://static.government.ru/media/files/60vyjG86YdZLNbGn4s0HYIBhov0cbG0r.pdf

6 Voir l'exposé des motifs du projet de l'arrêté gouvernemental sur les montants minima et maxima d'allocation chômage en 2019 (en russe).

http://www.consultant.ru/cons/cgi/online.cgi?req=doc; base=PNPA; $=39884 \# 02484067716157342$

7 Ibid. 
est écourtée par rapport à 2018 de six mois à trois mois pendant 12 mois calendaires consécutifs.

Force est donc de constater que les garanties supplémentaires d'appui social aux chômeurs de l'âge de la pré-retraite annoncées par le gouvernement se sont traduites par une légère augmentation du montant de l'indemnisation pour $41 \%{ }^{8}$ d'entre eux et par une aggravation de la situation pour tous les autres chômeurs. II faut noter que la Russie ne dispose pas d'un système d'assurance chômage qui repose sur des cotisations obligatoires, les allocations sont financées par le budget d'Etat. De ce fait, pour augmenter le montant de l'indemnité, le gouvernement doit réduire la durée d'indemnisation.

\section{II - LES MESURES SUPPLÉMENTAIRES D'AIDE À L'EMPLOI DES SENIORS}

La Loi n³52-FZ entrée en vigueur le 14 octobre 2018 criminalise le licenciement et le non-recrutement injustifiés de personnes de l'âge de la pré-retraite. Ladite loi a introduit un nouvel article 144.1 dans le Code pénal de la Fédération de la Russie qui prévoit des sanctions pour licenciement et non-recrutement abusifs fondés sur l'âge de la pré-retraite d'un salarié. Le dirigeant d'une entreprise ou un entrepreneur qui commet cette infraction risque une amende pouvant aller jusqu'à 200000 roubles (environ 2500 euros) ou jusqu'à l'équivalent de ses 18 salaires mensuels ou d'un travail d'intérêt général d'un maximum de 360 heures.

Ce dispositif qui a suscité une grande polémique reste toujours sujet à caution. D'après un récent sondage effectué sur Internet ${ }^{9}, 79 \%$ des visiteurs du site ne croient pas à l'efficacité de cette mesure. En effet, dans le Code pénal existent déjà des sanctions pour discrimination, dont une amende pouvant aller jusqu'à 300000 roubles. Mais les exemples de sanctions données ne sont pas légion. Les statistiques officielles de jurisprudence sur la discrimination au travail n'existent pas. Dans la plupart de cas, les licenciés et non-recrutés pour discrimination préfèrent tenter leur chance auprès d'autres employeurs plutôt que demander justice. Prouver l'acte discriminatoire est presque impossible puisque les employeurs évoquent rarement des motifs discriminatoires dans leurs documents officieux.

Dans ces circonstances, il apparaît que cette mesure ne peut vraiment pas empêcher les employeurs de licencier des seniors. D'ailleurs, il est fort probable que les personnes approchant l'âge de la pré-retraite éprouvent beaucoup plus de difficultés qu'avant pour trouver un travail et le conserver, parce que certaines entreprises seront plus prudentes dans le choix des salariés. Elles remplaceront ceux qui s'approcheront de cet âge.

Ainsi, la réforme et les mesures qu'elle contient visant à aider les personnes arrivant à l'âge de la pré-retraite peuvent conduire à la précarité la plupart d'entre eux, voire les plus jeunes.

8 Selon les estimations du Ministère du travail, environ $41 \%$ de chômeurs de l'âge de la préretraite percevront l'allocation maximale en 2019 (voir l'exposé des motifs du projet de l'arrêté gouvernemental sur les montants minimaux et maximaux d'allocation chômage en 2019).

9 Voir : Опрос: уголовная ответственность за увольнение предпенсионеров. Надо ^и вводить уголовную ответственность за увольнение работников предпенсионного возраста? https:// buh.ru/news/uchet_nalogi/73924/ 